\title{
ON RATIONAL POINTS ON CONICS
}

\author{
R. E. MACRAE
}

\begin{abstract}
The purpose of this paper is to prove the following result: let $K$ be a finitely, separably generated extension field of transcendence degree one and genus zero over the exact constant field $k$. Assume that $K$ has no $k$-rational points. Let $L$ be a subfield of $K$ that contains $k$. Then $L$ has a $k$-rational point if and only if $[K: L]$ is even.
\end{abstract}

1. Introduction. Let $k$ be an arbitrary field and $K$ be a function field in one variable over $k$ whose exact constant field is $k$. Moreover let $L$ be a subfield of $K$ which contains $k$ as a proper subfield. $L$ is also, of course, a function field in one variable with exact constant field $k$. An interesting question is the following: given that $K$ has no points rational over $k$ can we determine whether $L$ has any such points? The general question is a delicate and unsolved diophantine problem. However, progress can be made in certain special cases. See for example [2], [3], [4]. It is the purpose of this note to take care of the case in which the genus of $K$ is zero. The answer is, perhaps, a bit surprising and we refer the reader to the statement of Theorem A.

The author would like to thank M. Fried for asking the original question that is answered here.

2. Main result. Let $k, K, L$ be as in the first section. One knows that $K$ can be defined by a conic over $k$. See, for example, [1, Chapter III, §3.5]. Consequently, $K$ always has a point rational over some quadratic extension $k_{1}=k(e)$. We will make the additional assumption that $k_{1}$ is separable over $k$ and normalize matters so that $e^{2}=\varepsilon$ when the characteristic is not two and $e^{2}+e=\varepsilon$ otherwise. Denote by $\sigma$ the involution of $k_{1}$ that leaves $k$ fixed. We will usually write $a^{\sigma}=\bar{a}$.

THEOREM A. Let $K$ be a finitely, separably generated extension field of transcendence degree one and genus zero over the exact constant field $k$. Assume that $K$ has no $k$-rational points. Let $L$ be a subfield of $K$ that contains $k$. Then $L$ has a $k$-rational point if and only if $[K: L]$ is even.

Proof. Consider first $K_{1}=k_{1} \otimes_{k} K$. This is a field since $k$ is exact in $K$. Moreover, since the genus can only decline in ground field extension, $K_{1}$ is a field of genus zero with a $k_{1}$-rational point by hypothesis. Hence $K_{1}=k_{1}(t)$ is the field of rational functions in one variable over $k_{1}$. Let us denote by $\sigma$ also

Received by the editors September 17, 1976.

AMS (MOS) subject classifications (1970). Primary 14H05, 15G05.

C. American Mathematical Society 1977 
the involution of $k_{1}(t)$ that leaves $k(t)$ fixed. Since $k_{1}(t)=k_{1} \otimes_{k} K$ one can write the involution of $k_{1}(t)$ that leaves $K$ fixed in the form $\sigma A$ where $A$ is a $2 \times 2$ nonsingular matrix with entries in $k_{1}$. Since $(\sigma A)^{2}$ acts like the identity on $k_{1}$ we can write $(\sigma A)^{2}=g I$ for some $g$ in $k_{1}$. However $(\sigma A)^{2}=\sigma^{2} \bar{A} A=$ $\bar{A} A$. Putting the equations together and setting $A=\left(\begin{array}{ll}a & b \\ c & d\end{array}\right)$, we may write

$$
\left(\begin{array}{ll}
\bar{a} & \bar{b} \\
\bar{c} & \bar{d}
\end{array}\right)=\frac{g}{\Delta}\left(\begin{array}{cc}
d & -b \\
-c & a
\end{array}\right)
$$

where $\Delta=a d-b c$. Since $\Delta \neq 0$ an equating of coefficients in the above matrix equation shows that $N_{k_{1} / k}(g / \Delta)=1$. Hence by Hilbert's Theorem 90, $g / \Delta=f / \bar{f}$ for some $f$ in $k_{1}$. Let $B=f A$. Clearly $\sigma A$ and $\sigma B$ act in the same way on $k_{1}(t)$. We may assume from the start, therefore, that $A=\left(\begin{array}{ll}a & b \\ c & d\end{array}\right)$ and

$$
\left(\begin{array}{ll}
\bar{a} & \bar{b} \\
\bar{c} & \bar{d}
\end{array}\right)=\left(\begin{array}{cc}
d & -b \\
-c & a
\end{array}\right) .
$$

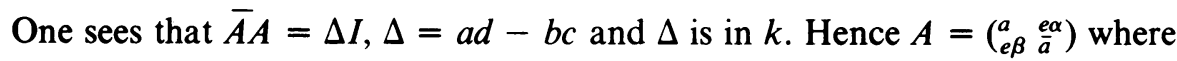
$\alpha$ and $\beta$ are in $k$ when the characteristic of $k$ is not two. (Recall that we had $k_{1}=k(e)$ with $e^{2}=\varepsilon$.) When the characteristic of $k$ is two we have $A=\left(\begin{array}{cc}a & \alpha \\ \beta & a\end{array}\right)$ with $\alpha$ and $\beta$ in $k$. Now $K_{1}=K(e)$ so there are elements $x$ and $y$ in $K$ such that $t=x+e y$ and $K=k(x, y)$. Let us apply $\sigma A$ to $t$. We obtain

$$
(a(x+e y)+e \alpha) /(e \beta(x+e y)+\bar{a})=x-e y .
$$

Hence

$$
e \beta\left(x^{2}-\varepsilon y^{2}\right)+\bar{a}(x-e y)-a(x+e y)-e \alpha=0 .
$$

Now write $a=\xi+e \eta$ for suitable $\xi, \eta$ in $k$. We get

$$
e \beta\left(x^{2}-\varepsilon y^{2}\right)=2 e(\eta x+\xi y)-e \alpha=0 .
$$

If we cancel $e$ and complete squares we get $(\beta \varepsilon y+\xi)^{2}-\varepsilon(\beta x-\eta)^{2}=\Delta$. By an obvious change of variable we may assume from the start that $y^{2}-\varepsilon x^{2}=$ $\Delta$ and $A=\left(\begin{array}{ll}0 & \Delta \\ 1 & 0\end{array}\right)$. Clearly $K$ has a $k$-rational point if and only if $\Delta$ is in the norm group $N k_{1}^{*}$. We remark at this point that a similar formula holds in characteristic two by using the Artin-Schreier equation rather than the square root. The computation is left to the reader. We next look at the subfield $L$ and set $L_{1}=k_{1} \otimes_{k} L$. Clearly $L_{1}$ has a $k_{1}$-rational point since $K_{1}$ is assumed to have such. Thus $L_{1}=k_{1}(u)$ where $u$ is a rational function of $t$. Observe that $u(\Delta / t)=u(t)^{\sigma A}=(a u+b) /(c u+d)$ for suitable $a, b, c, d$ in $k_{1}$. We may, however, change the variable $u$ as in the first part of the proof and assume from the start that $\bar{u}(\Delta / t)=\Delta^{\prime} / u$ for some $\Delta^{\prime}$ in $k$. As before $u=\bar{x}+e \bar{y}$ with $\bar{x}$ and $\bar{y}$ in $L, L=k(\bar{x}, \bar{y})$ and $\bar{y}^{2}-\varepsilon \bar{x}^{2}=\Delta^{\prime}$. Hence $L$ has a $k$-rational point if and only if $\Delta^{\prime}$ is in $N k_{1}^{*}$. Let us now relate $\Delta^{\prime}$ to $\Delta$. Write $u(t)=a t^{r} f(t) / g(t)$, where $a \in k_{1}, r$ is an integer, $f(t)$ and $g(t)$ are monic polynomials and neither vanish at zero. By hypothesis 


$$
\bar{a} \frac{\Delta^{r}}{t^{r}} \frac{\bar{f}(\Delta / t)}{\bar{g}(\Delta / t)}=\frac{\Delta^{\prime}}{a t^{r}} \frac{g(t)}{f(t)} .
$$

From this it follows easily that the degrees of $f$ and $g$ are equal. Next let $a_{0}$ and $b_{0}$ be the constant terms in $f$ and $g$, respectively. Then

$$
a \bar{a} \Delta^{r} \frac{\bar{a}_{0} \hat{\bar{f}}(t)}{\bar{b}_{0} \hat{\bar{g}}(t)}=\Delta^{\prime} \frac{g(t)}{f(t)}
$$

where $\hat{\bar{f}}$ and $\hat{\bar{g}}$ are monic. Hence $a \bar{a} \Delta^{r} \bar{a}_{0} / \bar{b}_{0}=\Delta^{\prime}, \hat{\bar{f}}(t)=g(t)$ and $\hat{\bar{g}}(t)=f(t)$. From the latter equations we see that $\Delta^{m} / \bar{a}_{0}=b_{0}$ and $\Delta^{m} / \bar{b}_{0}=a_{0}$, where $m$ is the common degree of $f$ and $g$. Since $\Delta$ is in $k$ we see that $a_{0} / b_{0}=\bar{a}_{0} / \bar{b}_{0}$. Putting all this together shows that $\Delta^{\prime}=\Delta^{m+r} N\left(a / b_{0}\right)$ and $\Delta^{\prime}=$ $\Delta^{r-m} N\left(a / a_{0}\right)$. Since one of the two numbers $|m+r|$ and $|r-m|$ equals $\left[k_{1}(t): k_{1}(u)\right]=[K: L]$, we have proved our result. We should remark that the computations in characteristic two are a bit different but the reader will have no difficulty in carrying them out.

3. Final observations. There is one rather interesting special case of Theorem A that we would like to mention here. Let $k$ be the real field. The Riemann surface for $K$ can be given the structure of a closed 2-manifold (called a Klein surface) which may have a boundary and may be nonorientable. In the case of genus zero the two possible cases are (i) a closed disc and (ii) the projective plane. The two cases are distinguished by, respectively, whether there are or are not real points on the conic which describe the surface. Now then, the situation $L \leqslant K,[K: L]=n$ is described by a rational map of degree $n$ of the projective plane onto either a closed disc or another projective plane. If we exclude the branch points of this map, we get an ordinary covering of either the cylinder or Möbius strip by another Möbius strip. Theorem A then states that the covered surface is a Möbius strip if and only if $n$ is odd. It is possible (although, perhaps, irrelevant) that the map can be realized physically by paper and paste and the reason that even and odd $n$ enter the picture will appear in a very obvious way. The reader is invited to try the experiment!

\section{REFERENCES}

1. M. Eichler, Introduction to the theory of algebraic numbers and functions, Academic Press, New York, 1966.

2. M. Fried, Brauer groups and Jacobians (preprint).

3. __ Poncelet correspondences, finite correspondences and Ritt's theorem on commuting morphisms (preprint).

4. R. E. MacRae, On the two-sheeted coverings of conics by elliptic curves, Trans. Amer. Math. Soc. 211 (1975), 277-287.

Department of Mathematics, University of Colorado, Boulder, Colorado 80302 